\title{
Continuous-Time Mean-Variance Portfolio Selection Problem with Ho-Lee Stochastic Interest Rates
}

\author{
Chuangwei $\operatorname{Lin}^{1}$ and Li Zeng ${ }^{2, *}$ \\ ${ }^{1}$ Research Center for International Trade and Economic, Guangdong University of Foreign Studies, GuangZhou, Guangdong \\ 510006, P. R. China \\ ${ }^{2}$ School of Finance, Guangdong University of Foreign Studies, GuangZhou, Guangdong 510006, P. R. China \\ ${ }^{*}$ Corresponding author
}

\begin{abstract}
This paper investigates a continuous-time meanvariance portfolio selection problem with Ho-Lee stochastic interest rates. Compared with the mean-variance model with deterministic interest rate, a verification theorem without the classical Lipschitz and growth conditions is required to solve our portfolio selection problem. The optimal investment strategy, the value function and the efficient frontier are derived in closedform. Some existing results are obtained as special cases in our paper.
\end{abstract}

Keywords-mean-variance portfolio selection; Ho-Lee stochastic interest rate; efficient frontier; stochastic control.

\section{INTRODUCTION}

After the pioneering work by [1] in the single-period mean-variance formulation, the mean-variance portfolio selection problem has provided a fundamental basis for portfolio construction and has stimulated hundreds of extensions and applications. References [2] and [3] are the first to extend the original static mean-variance model to multi-period and continuous-time cases respectively. Since then, various kinds of problems under mean-variance criterion have been investigated analytically. For instances, [4] and [5] considered an asset and liability management problem. References [6] and [7] solved a portfolio selection problem when stock prices follow jump diffusion processes. References [8] and [9] studied mean-variance optimization problems for an insurer. Reference [10] investigated mean-variance portfolio optimization problems with no-shorting constraint under discrete-time settings.

However, most existing literatures deal with the continuous-time mean-variance optimization problem under the assumption that the interest rate is deterministic. But in the real world, the interest rate is affected greatly by some uncertain affairs, such as inflation, war and disaster. Therefore, it is more realistic to assume that the interest rate is a stochastic process rather than a deterministic one. Stochastic interest rate models are widely applied in option pricing, hedging and portfolio optimization. In the past years, many scholars have devoted themselves to the optimal portfolio selection problem with stochastic interest rate. For examples, [11] considered an investment problem to maximize the power-utility from terminal wealth under the assumption that the interest rate is a stochastic process. [12] solved a powerutility maximization problem with Heston's stochastic volatility and presented a verification result which covers Heston's stochastic volatility model. [13] extended the previous work of the optimal management of an aggregated pension fund of defined benefit type by incorporating a more realistic assumption that the interest rate is stochastic. [14] considered a mean-variance optimization problem with stochastic interest rate described by extended CIR model via linear-quadratic techniques. [15] considered an optimal assetliability management problem with stochastic interest rates and inflation risks under the mean-variance framework. Unfortunately, the assumption of uniform bounded coefficients does not include the case of Ho-Lee stochastic interest model. [16] considered the portfolio selection problems with regime switching, where the coefficients, including the interest rate, depend on the market states and hence have some randomness since the market states in the future are uncertain. For this reason, portfolio optimization problems with regime switching can be classified as problems with stochastic interest rate. However, when given all possible market states in the future, the coefficients become continuous bounded functions, hence we can solve these problems with regime switching by using the usual Lipschitz conditions and classic verification theorem.

Overall, mean-variance portfolio selection models with stochastic interest rates are limited. Our paper will focus on this topic and considers a mean-variance portfolio selection problem with Ho-Lee stochastic interest rate. The contributions of our paper are as follows: (a) Present a new definition of admissible strategies and a new sufficient condition that guarantee a candidate for the value function to be indeed optimal; (b) by constructing an auxiliary variable, derive the analytic solution of the wealth process corresponding to a candidate for the optimal strategy; (c) analyze at length the properties of candidates for the optimal strategy and the value function; (d) obtain the analytic expressions of the optimal strategy and the value function which do not depend on other unknown functions; $(e)$ the ideas and methods in this paper may throw some lights on mean-variance problems with other stochastic interest rates or stochastic volatility rates.

The rest of this paper is organized as follows. In Section 2, we formulate the mean-variance portfolio selection model with Ho-Lee stochastic interest rates. In Section 3, we obtain the candidates for the optimal strategy and the value function 
in Ho-Lee model. In Section 4, we analyze in detail the properties of these candidates. We obtain the efficient frontier in closed-form in Section 5. Section 6 concludes this paper.

\section{PROBLEM FORMULATION}

Let $\left(\Omega, \mathcal{F}, P,\left\{\mathcal{F}_{t}\right\}_{t \geq 0}\right)$ be a fixed filtered complete probability space on which is defined a standard $\mathcal{F}_{t}$-adapted Brownian motion $W(t)$, here $\mathcal{F}_{t}=\sigma(W(s): 0 \leq s \leq t)$. An investor joins a market at time 0 with initial wealth $x_{0}>0$ and plans to invest dynamically over a fixed time horizon $T$. For the sake of simplicity, we assume that there are two assets traded in a frictionless market. One asset is a saving account whose price process is

$$
d B(t)=B(t) r(t) d t, B(0)=b_{0},
$$

Where $d$ hereafter represents the differential operator. Different from the model with constant interest rate, here the short rate $r(t)$ follows the differential equation

$$
d r(t)=(\tilde{a}(t)+b \xi(t)) d t+b d W(t), r(0)=r_{0},
$$

where $\tilde{a}(t)$ is a deterministic and continuously differentiable function, $b>0$ is a constant, the risk premium $\xi(t)$ is a deterministic and continuous function, $W(t)$ is a standard Brownian motion. The interested readers can refer to [17] for detailed information. The other asset is a (zero) bond whose price process is modeled as

$$
d S(t)=S(t)[(r(t)+\xi(t) \sigma(t)) d t+\sigma(t) d W(t)], S(0)=s_{0}
$$

where $\sigma(t)$ is a deterministic and continuously differentiable function. Here the appreciation rate is $r(t)+\xi(t) \sigma(t):=$

$\mu(t)$, which results from the fact that the risk premium $\xi(t)$ is defined as $\frac{\mu(t)-r(t)}{\sigma(t)}$. The interested reader can refer to [11] (p.3) for more information.

Denote by $\pi(t)$ the amount invested in the (zero) bond at time $t \quad(t \in[0, T])$ and by $X^{\pi}(t)$ the wealth at time $t$ corresponding to investment strategy $\pi$. Then the wealth process follows the following differential equation:

$$
\begin{aligned}
& d X^{\pi}(t) \\
= & \pi(t)[(r(t)+\xi(t) \sigma(t)) d t+\sigma(t) d W(t)] \\
& +\left(\mathrm{X}^{\pi}(t)-\pi(t)\right) r(t) d t \\
= & {\left[\pi(t) \xi(t) \sigma(t)+\mathrm{X}^{\pi}(t) r(t)\right] d t+\pi(t) \sigma(t) d W(t) }
\end{aligned}
$$

with initial condition $X(0)=x_{0}$.

In this paper, we consider the following mean-variance portfolio selection problem

$$
P(w)\left\{\begin{array}{c}
\min _{\pi(\cdot) \in \Pi\left(0, x_{0}\right)} \mathrm{E}\left[X^{\pi}(T)-w\right]^{2} \\
\text { s.t. } \mathrm{E}\left(X^{\pi}(T)\right)=w
\end{array}\right.
$$

where $w$ is a pregiven return level, and $\Pi\left(0, x_{0}\right)$ denotes the set of all admissible controls defined as below.

Definition 1 A control $\pi(t)$ is said to be admissible if

(i) for any initial wealth $x_{0}>0$, the stochastic differential equation (SDE for short hereafter) (1) has a unique solution $X^{\pi}(\cdot)$ corresponding to $\pi(\cdot)$;

(ii) for all $k \in \mathbb{N}:=\{1,2, \cdots\}$, the corresponding solution $X^{\pi}(\cdot)$ satisfies

$$
\mathrm{E}\left(\sup _{t \in[0, T]}\left|X^{\pi}(t)\right|^{k}\right)<+\infty
$$

(iii) for all $k \in \mathbb{N}, \mathrm{E}\left(\int_{0}^{T}|\pi(t)|^{k} d t\right)<+\infty$.

By convex optimization theory, problem $P(w)$ can be solved via the following optimal stochastic control problem with a Lagrange multiplier $2 \lambda$ :

$$
P L 1(\lambda, w) \min _{\pi(\cdot) \in \Pi\left(0, x_{0}\right)}\left\{\mathrm{E}\left[X^{\pi}(T)-w\right]^{2}-2 \lambda\left[\mathrm{E}\left(X^{\pi}(T)\right)-w\right]\right\}
$$

The relationship between the optimal solutions of these two problems is concluded in the following lemma (see [18]).

Lemma 1 Denote by $\Gamma(\lambda)$ and $\{\hat{\pi}(\lambda, t, x(t)), t \in[0, T]\}$, respectively, the optimal value and the optimal strategy of problem $\operatorname{PL1}(\lambda, w)$. Then the optimal value and the optimal strategy of problem $P(w)$ are $\sup _{\lambda \in R} \Gamma(\lambda)$ and $\left\{\hat{\pi}\left(\lambda^{*}, t, x(t)\right)\right.$, $t \in[0, T]\}$ respectively, where $\lambda^{*}=\underset{\lambda \in R}{\arg \sup _{\lambda \in}} \Gamma(\lambda)$.

Since the objective function of problem $\operatorname{PL1}(\lambda, w)$ can be rewritten as $\mathrm{E}\left[X^{\pi}(T)-(\lambda+w)\right]^{2}-\lambda^{2}$, solving problem $\operatorname{PL1}(\lambda, w)$ is equivalent to solving problem

$$
P L 2(\lambda, w) \min _{\pi(\cdot) \in \Pi\left(0, x_{0}\right)} \mathrm{E}\left[X^{\pi}(T)-(\lambda+w)\right]^{2} .
$$

For problem $P L 2(\lambda, w)$, define the value function 


$$
\begin{array}{r}
V(t, x, r)=\min _{\pi(\cdot) \in \Pi\left(0, x_{0}\right)} \mathrm{E}\left[\left(X^{\pi}(T)-(\lambda+w)\right)^{2}\right. \\
\mid X(t)=x, r(t)=r] .
\end{array}
$$

By Bellman's optimality principle for dynamic programming, we have

$$
V(t, x, r)=\min _{\pi(s): t \leq s \leq t+h} \mathrm{E}\left[V\left(t+h, X^{\pi}(t+h), r(t+h)\right)\right],
$$

$$
(\forall h>0)
$$

Define an operator

$$
\begin{aligned}
& A^{\pi(t)} V(t, x, r) \\
= & V_{t}+V_{x}[\pi(t) \xi(t) \sigma(t)+x(t) r(t)]+\frac{1}{2} V_{x x} \pi^{2} \sigma^{2} \\
& +V_{x r} b \pi(t) \sigma(t)+\frac{1}{2} V_{r r} b^{2}+V_{r}(\tilde{a}(t)+b \xi(t)) .
\end{aligned}
$$

Then, applying the Itô's formula, we have

$$
\begin{aligned}
& V\left(t+h, X^{\pi}(t+h), r(t+h)\right) \\
= & V(t, x, h)+\int_{t}^{t+h} A^{\pi(s)} V(s, X(s), r(s)) d s \\
& +\int_{t}^{t+h}\left(b V_{r}+V_{x} \pi(s) \sigma(s)\right) d W(s)
\end{aligned}
$$

According to [19](p.139), if

$$
E_{t, x}\left[\int_{t}^{t+h}\left(b V_{r}+V_{x} \pi(s) \sigma(s)\right)^{2} d s\right]<+\infty
$$

then

$$
\int_{t}^{t+h}\left(b V_{r}+V_{x} \pi(s) \sigma(s)\right) d W(s)
$$

is a martingale. Therefore, when

$$
E_{t, x}\left[\int_{t}^{t+h}\left(b V_{r}+V_{x} \pi(s) \sigma(s)\right)^{2} d s\right]<+\infty
$$

substituting (3) into (2) yields the Hamilton-Jacob-Bellman (HJB for short) equation of $V(t, x, r)$ as follows:

$$
\begin{gathered}
V_{t}+V_{x} x r+\frac{1}{2} V_{r r} b^{2}+V_{r}(\tilde{a}(t)+b \xi(t))+ \\
\min _{\pi(t)}\left\{\frac{1}{2} V_{x x} \pi^{2} \sigma^{2}+V_{r x} b \pi(t) \sigma(t)+V_{x} \pi(t) \xi(t) \sigma(t)\right\}=0
\end{gathered}
$$

with the boundary condition $V(T, x, r)=(x-(\lambda+w))^{2}$.

We care about the contribution of HJB equation (4) to derive the optimal strategy and the value function. So we give the following verification theorem.

Theorem 1 Suppose that $v(t, x, r) \in C^{1,2,2}([0, T] \times O)$, where $O \subseteq R^{2}$, satisfies

(i) $v(t, x, r)$ solves (4) with boundary condition;

(ii) for any admissible control $\pi(\cdot)$ and its corresponding wealth process,

$$
E_{t, x}\left[\int_{t}^{t+h}\left(b V_{r}+V_{x} \pi(s) \sigma(s)\right)^{2} d s\right]<+\infty,
$$

for all $t \in[0, T], h>0$;

(iii) for all sequences of stopping times $\left\{\tau_{n}: 0 \leq \tau_{n} \leq T\right\}_{n \in \mathbb{N}}$ and any admissible strategy $\pi(\cdot) \in \Pi\left(0, x_{0}\right)$, the sequence $\left\{v\left(\tau_{n}, X^{\pi}\left(\tau_{n}\right), r\left(\tau_{n}\right)\right)\right\}_{n \in \mathbb{N}}$ is uniformly integrable.

Then we have

(a) $v(t, x, r) \leq V(t, x, r)$;

(b) if there exists an admissible strategy $\widehat{\pi}(t) \in$ $\arg \min _{\pi}\left\{A^{\pi} v\left(t, X^{\hat{\pi}}(t), r\right)\right\}$, then $v(t, x, r)=V(t, x, r)$.

Before solving our problem, we must explain why the definition of an admissible strategy and the conditions of verification theorem are so different to the traditional ones in [20]. In [20] (p.158), a system of stochastic differential equations is defined as the following form:

$$
d x=f(s, x(s), u(s)) d s+\sigma(s, x(s), u(s)) d w(s),
$$

where $u(s)$ is the control at time $s, w(s)$ is a Brownian motion, and $f$ and $\sigma$ satisfy Lipschitz and growth conditions

$$
\begin{gathered}
\left|f_{t}\right|+\left|f_{x}\right| \leq C, \quad\left|\sigma_{t}\right|+\left|\sigma_{x}\right| \leq C, \\
|f(s, x, v)| \leq C(1+|x|+|v|), \\
|\sigma(s, x, v)| \leq C(1+|x|+|v|),
\end{gathered}
$$


for some constant $C$.

Lipschitz and growth conditions play a key role in deriving the optimal solutions when the interest rate is deterministic:

- when (5)-(7) are satisfied, then (i) and (ii) in Definition 1 hold true naturally when (iii) in Definition 1 holds true;

- $\quad$ (7) can guarantee condition (ii) in Theorem 1 holding true;

- when $|v| \leq K\left(1+|x|^{k}\right)$ for suitable $K$ and $k \in \mathbb{N}$, condition (ii) in Definition 1 ensures uniform integrability of $v$.

It is easy to obtain $|v| \leq K\left(1+|x|^{k}\right)$ for the mean-variance model with deterministic interest rate. Hence, in the case of deterministic interest rate, when the Lipschitz and growth conditions hold, we just need condition (iii) of Definition (1) and condition (i) of Theorem (1).

Unfortunately, when the interest rate $r(t)$ is a stochastic process, the coefficients of wealth process (1) do not satisfy Lipschitz and growth conditions. So we need other conditions in the new definition of admissible strategies and the verification theorem. The new definition and the new verification theorem provide sufficient conditions to guarantee that the candidates for the optimal solutions are indeed optimal.

Next we set about deducing the optimal strategy and the value function of problem $P L 2(\lambda, w)$ by Theorem 1 .

\section{CANDIDATES FOR THE OPTIMAL SOLUTIONS}

Assume that $v$ is a solution of HJB equation (4). When $v_{x x}>0$, the candidate for the optimal strategy of problem $\operatorname{PL2}(\lambda, w)$ is

$$
\hat{\pi}(t)=-\frac{v_{r x} b+v_{x} \xi(t)}{v_{x x} \sigma(t)}
$$

Inserting equation (8) back into (4) yields

$$
\begin{aligned}
v_{t}+v_{x} x r+ & \frac{1}{2} v_{r r} b^{2}+v_{r}(\hat{a}(t)+b \xi(t)) \\
- & \frac{1}{2} \frac{\left(v_{r x} b+v_{x} \xi(t)\right)^{2}}{v_{x x}}=0
\end{aligned}
$$

with terminal condition $v(T, x, r)=(x-(\lambda+w))^{2}$.

We guess and subsequently verify that $v$ has the form

$$
v(t, x, r)=P(t, r) x^{2}-2(\lambda+w) Q(t, r) x+(\lambda+w)^{2} R(t, r)
$$

with $P(t, r)>0, P(T, r)=1, Q(T, r)=1$ and $R(T, r)=1$. Substituting the above expression into equation (9), we obtain

$$
\left[\begin{array}{c}
P_{t}(t, r)+(\tilde{a}(t)+b \xi(t)) P_{r}(t, r)+2 r P(t, r) \\
-\frac{\left(b P_{t}(t, r)+\xi(t) P(t, r)\right)^{2}}{P(t, r)}+\frac{1}{2} b^{2} P_{r r}
\end{array}\right] x^{2}-2(\lambda+w) x
$$

$$
\begin{gathered}
{\left[\begin{array}{c}
Q_{t}(t, r)+r Q(t, r)+(\tilde{a}(t)+b \xi(t)) Q_{r}(t, r) \\
-\frac{\left(b P_{r}(t, r)+\xi(t) P(t, r)\right)\left(b Q_{r}(t, r)+\xi(t) Q(t, r)\right)}{P(t, r)}+\frac{1}{2} b^{2} Q_{r r}
\end{array}\right]} \\
+(\lambda+w)^{2}\left[\begin{array}{c}
R_{t}(t, r)+(\tilde{a}(t)+b \xi(t)) R_{r}(t, r)+\frac{1}{2} b^{2} R_{r r}(t, r) \\
-\frac{\left(b Q_{r}(t, r)+\xi(t) Q(t, r)\right)^{2}}{P(t, r)}
\end{array}\right]
\end{gathered}
$$

$=0$

This leads to the following partial differential equations for $P(t, r), Q(t, r)$ and $R(t, r)$ respectively:

$$
\begin{gathered}
\left\{\begin{array}{l}
P_{t}+(\tilde{a}+b \xi(t)) P_{r}+2 r P-\frac{\left(\xi(t) P+b P_{r}(t, r)\right)^{2}}{P} \\
+\frac{1}{2} b^{2} P_{r r}=0, \\
P(t, r)>0, P(T, r)=1 ;
\end{array}\right. \\
\left\{\begin{array}{l}
Q_{t}+r Q+(\tilde{a}+b \xi(t)) Q_{r} \\
-\frac{\left(\xi(t) P+b P_{r}\right)\left(\xi(t) Q+b Q_{r}\right)}{P}+\frac{1}{2} b^{2} Q_{r r}=0, \\
Q(T, r)=1 ;
\end{array}\right. \\
\left\{\begin{array}{c}
R_{t}(t, r)+(\tilde{a}+b \xi(t)) R_{r}(t, r)+\frac{1}{2} b^{2} R_{r r}(t, r) \\
-\frac{\left(\xi(t) Q+b Q_{r}\right)^{2}}{P}=0, \\
R(T, r)=1 .
\end{array}\right.
\end{gathered}
$$

Let $P(t, r)$ and $Q(t, r)$ be of the forms:

$$
\begin{aligned}
& P(t, r)=e^{A(t) r+B(t)} \\
& Q(t, r)=e^{C(t) r+D(t)}
\end{aligned}
$$

with terminal conditions

$$
A(T)=B(T)=C(T)=D(t)=0 .
$$

Inserting (13) into (10) gives 


$$
\begin{aligned}
& \left(2+A^{\prime}(t)\right) r+B^{\prime}(t)+(\tilde{a}-b \xi(t)) A(t) \\
& -\frac{1}{2} b^{2} A^{2}(t)-\xi^{2}(t)=0 .
\end{aligned}
$$

Substituting (14) into (11) results in

$$
\begin{aligned}
& \left(1+C^{\prime}(t)\right) r+D^{\prime}(t)+\tilde{a} C(t) \\
& -(\xi(t)+b C(t))^{2}-\frac{1}{2} b^{2} C^{2}(t)=0 .
\end{aligned}
$$

Solving these two equations together with terminal conditions (15) yields

$$
\begin{gathered}
A(t)=2(T-t)=2 C(t), \\
B(t)=\int_{t}^{T}[(\tilde{a}(s)-b \xi(s)) A(s) \\
\left.-\frac{1}{2} b^{2} A^{2}(s)-\xi^{2}(s)\right] d s
\end{gathered}
$$

and

$$
\begin{aligned}
D(t)= & \int_{t}^{T}\left[\tilde{a}(s) C(s)-(\xi(s)+b C(s))^{2}\right. \\
& \left.-\frac{1}{2} b^{2} C^{2}(s)\right] d s .
\end{aligned}
$$

Noting that $A(t)=2 C(t)$, it is easy to find that

$$
2 D(t)-B(t)=-\int_{t}^{T}(\xi(s)+b C(s))^{2} d s
$$

After adopting the expressions of $P(t, r)$ and $Q(t, r)$, (12) becomes

$$
\begin{aligned}
& R_{t}(t, r)+(\tilde{a}+b \xi(t)) R_{r}(t, r)+\frac{1}{2} b^{2} R_{r r}(t, r) \\
& -(\xi(t)+b C(t))^{2} e^{2 D(t)-B(t)}=0 .
\end{aligned}
$$

Noting (19), the solution of the above equation can be of the form

$$
R(t)=e^{2 D(t)-B(t)}=e^{-\int_{t}^{T}(\xi(s)+b C(s))^{2} d s}
$$

The candidate for the optimal strategy has the form

$$
\begin{aligned}
\hat{\pi}(t, x)= & \frac{(\lambda+w)(\xi(t)+b C(t))}{\sigma(t)} e^{-C(t) r+D(t)-B(t)} \\
& -\frac{\xi(t)+b A(t)}{\sigma(t)} x(t)
\end{aligned}
$$

and the respective candidate for the value function is of the form

$$
\begin{aligned}
v(t, x, r)= & e^{A(t) r+B(t)} x^{2}-2(\lambda+w) e^{C(t) r+D(t)} x \\
& +(\lambda+w)^{2} e^{-\int_{t}^{T}(\xi(s)+b C(s))^{2} d s}
\end{aligned}
$$

with different expressions of $A(t), B(t), C(t)$, and $D(t)$ given above. In the next section, we will take effort to analyze the properties of the candidates $\hat{\pi}(t, x)$ and $v$.

\section{PROPERTIES OF CANDIDATES $\hat{\pi}(t, x)$ AND $v$}

In order to analyze the properties of the candidates, we first solve the SDE of $r(t)$ and introduce some relevant lemmas.

In Ho-Lee model, the short rate $r(t)$ is

$$
r(t)=r_{0}+\int_{0}^{t}(\tilde{a}(s)+b \xi(s)) d s+\int_{0}^{t} b d W(s)
$$

Lemma $2 \mathrm{E}\left(\sup _{t \in[0, T]} e^{\int_{0}^{t} h(s) d W(s)}\right)<+\infty$, for any deterministic and bounded function $h(t)$.

$$
\text { Lemma } 3 \int_{0}^{T}|r(t)| d t<+\infty \text { a.s. . }
$$

Lemma 4 For any integer $m$,

$$
\mathrm{E}\left(\sup _{t \in[0, T]} e^{2 m \int_{0}^{t} r(s) d s}\right)<+\infty \text {. }
$$

Now we set about analyzing the properties of $\hat{\pi}(t, x)$ and $v$. By using Lemma 1-Lemma 4, we can prove the following theorems: Theorem 2 and Theorem 3.

Theorem $2 \hat{\pi}(t, x)$ in (22) is admissible.

Theorem 3 The candidate $v$ for value function satisfies all the conditions in Theorem 1.

By the previous analysis, we have proved that the candidates for the optimal solutions are indeed optimal. Thus, the optimal strategy and the value function of $\operatorname{PL2}(\lambda, w)$ are concluded in the following theorem.

Theorem 4 For problem $P L 2(\lambda, w)$, the optimal strategy is 


$$
\begin{aligned}
\hat{\pi}(t, x(t))= & \frac{(\lambda+w)(\xi(t)+b C(t))}{\sigma(t)} e^{-C(t) r(t)+D(t)-B(t)} \\
& -\frac{\xi(t)+b A(t)}{\sigma(t)} x(t), \quad t \in[0, T]
\end{aligned}
$$

and the value function is

$$
\begin{aligned}
V(t, x, r)= & e^{A(t) r(t)+B(t)} x^{2}-2(\lambda+w) e^{C(t) r(t)+D(t)} x \\
& +(\lambda+w)^{2} e^{-\int_{t}^{T}(b C(s)+\xi(s))^{2} d s}, t \in[0, T],
\end{aligned}
$$

where $A(t), B(t), C(t)$ and $D(t)$ are given by (16)-(18) for Ho-Lee model.

\section{OPtIMAL SOLUTIONS AND EFFICIENT FRONTIER OF PROBLEM $P(w)$}

According to the relationship between $P L 1(\lambda, w)$ and $P L 2(\lambda, w)$, if we define $\Gamma(\lambda)$ as the optimal objective function of $\operatorname{PL1}(\lambda, w)$, then

$$
\begin{aligned}
\Gamma(\lambda)= & P\left(0, r_{0}\right) x_{0}^{2}-2 w Q\left(0, r_{0}\right) x_{0}+w^{2} R(0) \\
& +\lambda^{2}(R(0)-1)+2 \lambda\left(w R(0)-Q\left(0, r_{0}\right) x_{0}\right)
\end{aligned}
$$

$$
\begin{aligned}
& \text { Noting that } R(0)=e^{-\int_{0}^{T}(b C(s)+\xi(s))^{2} d s}<1, \text { then } \\
& \lambda^{*}=\arg \left(\max _{\lambda \in R} \Gamma(\lambda)\right) \text { exists and is }
\end{aligned}
$$

$$
\lambda^{*}=\frac{w R(0)-Q\left(0, r_{0}\right) x_{0}}{1-R(0)}
$$

Now according to the relationship between $P(w)$ and $P L 1(\lambda, w)$ in Lemma 1, the optimal strategy and the efficient

frontier of problem $P(w)$ are concluded in the theorem below.

Theorem 5 For problem $P(w)$, the optimal strategy is

$$
\begin{aligned}
\pi^{*}(t, x(t))= & \left.\hat{\pi}(t, x(t))\right|_{\lambda=\lambda^{*}} \\
= & \frac{w-Q\left(0, r_{0}\right) x_{0}}{1-R(0)} \frac{b C(t)+\xi(t)}{\sigma(t)} \frac{Q(t, r)}{P(t, r)} \\
& -\frac{b A(t)+\xi(t)}{\sigma(t)} x(t),
\end{aligned}
$$

and the efficient frontier is

$$
\operatorname{Var}(X(T))=\frac{R(0)}{1-R(0)}\left[\mathrm{E}(X(T))-\frac{Q\left(0, r_{0}\right)}{R(0)} x_{0}\right]^{2}
$$

where $R(0)=e^{-\int_{0}^{T}(\xi(s)+b C(s))^{2} d s}$.

Proof: Insert $\lambda^{*}$ into $\hat{\pi}(t, x(t))$ and $\Gamma(\lambda)$ respectively and simplify them. Then we will obtain the desired results.

\section{CONCLUSIONS}

In this paper, a continuous-time mean-variance portfolio model with stochastic interest rates has been studied. The assumption of stochastic interest rates is more realistic but results in difficulties for handling the problem. In contrast to the model with a deterministic interest rate, our wealth process does not satisfy the classical Lipschitz and growth conditions. Hence we require a new definition of admissible strategy and a new verification theorem that does not depend on the classical Lipschitz and growth conditions. Further, different from the existing power-utility problems with stochastic interest rate, our wealth process corresponding to a candidate for the optimal strategy does not follow a homogeneous differential equation any more, which leads to more difficulties in deriving the analytic solution of the wealth process. We tackle this problem by constructing an auxiliary variable and deriving its analytic expression. Our efficient frontier coincides with that in [3] when the stochastic interest rate is reduced to a deterministic one. The ideas and methods in this paper may throw some lights on mean-variance problems with other stochastic interest rates or stochastic volatility rates, such as Heston stochastic volatility, CIR interest rate, etc.

\section{ACKNOWLEDGMENTS}

This research is supported by Guangdong Philosophy and Social Science Planning Project (No. GD13XGL41) and Innovative School Project in Higher Education of Guangdong, China (No. GWTP-SY-2014-02).

\section{REFERENCES}

[1] H. Markowitz, "Portfolio selection," Journal of Finance, vol. 7, pp. 7791, 1952.

[2] D. Li and W. L. Ng, "Optimal dynamic portfolio selection: multiperiod mean-variance formulation,” Mathematical Finance, vol. 10, pp. 387406, 2000.

[3] X. Y. Zhou and D. Li, "Continuous-time mean-variance portfolio selection: a stochastic LQ framework," Applied Mathematics and Optimization, vol. 42, pp. 19-33, 2000.

[4] M. C. Chiu and D. Li, "Asset and liability management under a continuous-time mean-variance optimization framework," Insurance: Mathematics and Economics, vol. 39, pp. 330-355, 2006.

[5] S. X. Xie, Z. F. Li and S. Y. Wang, "Continuous-time portfolio selection with liability: mean-variance model and stochastic LQ approach," Insurance: Mathematics and Economics, vol. 42, pp. 943-953, 2008.

[6] S. Alp Ö and R. Korn, "Continuous-time mean-variance portfolio optimization in a jump-diffusion market," Decisions in Economics and Finance, vol. 34, pp. 21-40, 2011.

[7] Y. Zeng, Z. F. Li, and H. L. Wu, "Optimal portfolio selection in a Lévy market with unconstrolled cash flow and only risky assets,” International Journal of Control, vol. 86, pp. 426-437, 2013.

[8] N. Bäuerle, "Benchmark and mean-variance problems for insurers," Mathematical Methods of Operations Research, vol. 62, pp. 159-165 , 2005. 
[9] Y. Zeng and Z. F. Li, "Optimal time-consistent investment and reinsurance policies for mean-variance insurers," Insurance: Mathematics and Economics, vol. 49, pp. 145-154, 2011.

[10] X. Li, X. Y. Zhou and A. E. B. Lim, "Dynamic mean-variance portfolio selection with no-shorting constraints," SIAM Journal on Control and Optimization, vol. 40, pp. 1540-1555, 2002.

[11] R. Korn and H. Kraft, "A stochastic control approach to portfolio problems with stochastic interest rates," SIAM Journal on Control and Optimization, vol. 40, pp. 1250-1269, 2001.

[12] H. Kraft, "Optimal portfolios and Heston's stochastic volatility model: an explicit solution,” Quantitative Finance, vol. 5, pp. 303-313, 2005.

[13] R. Josa-Fombellida and J. P. Rincón-Zapatero, “Optimal asset allocation for aggregated defined benefit pension funds with stochastic interest rates," European Journal of Operational Research, vol. 201, pp. 211221, 2010.

[14] K. Ferland and F. Watier, "Mean-variance efficiency with extended CIR interest rates,” Appliced Stochastic Models in Business and Industry, vol. 26, pp.71-84, 2010.

[15] J. P. Pan and Q. X. Xiao, "Optimal mean-variance asset-liability management with stochastic interest rates and inflation risks," Mathematical Methods of Operations Research, vol. 85, pp. 491-519 , 2017.

[16] X. Y. Zhou and G. Yin, "Markowitz's mean-variance portfolio selection with regime switching: a continous-time model," SIAM Journal on Control and Optimization, vol. 42, pp. 1466-1482, 2003.

[17] M. Musiela and M. Rutkowski, Martingale methods in financial modelling, New York: Springer Berlin Heidelberg, 1997.

[18] D. G. Luenberger, Optimization by vector space methods, New York : Wiley, 1968.

[19] R. Daniel and Y. Marc, Continuous martingales and brownian motion. New York: Springer Berlin Heidelberg, 2005.

[20] W. H. Fleming and H. M. Soner, Controlled Markov processes and viscosity solutions, New York: Springer Berlin Heidelberg, 1993. 